Journal section: Oral Medicine and Pathology

Publication Types: Case Report doi:10.4317/medoral.16.e11

http://dx.doi.org/doi:10.4317/medoral.16.e11

\title{
Oral postinflammatory pigmentation: An analysis of 7 cases
}

\author{
Giovanni Mergoni ${ }^{1}$, Sertan Ergun ${ }^{2}$, Paolo Vescovi ${ }^{3}$, Özgür Mete ${ }^{4}$, Hakkı Tanyeri $^{5}$, Marco Meleti ${ }^{6}$
}

\begin{abstract}
${ }^{1}$ Resident, Unit of Oral Pathology, Oral Medicine and Laser-assisted Oral Surgery, Section of Odontostomatology, Department of Otolaryngological/Dental/Ophthalmological and Cervico-Facial Sciences, University of Parma, Italy

${ }^{2}$ Research Assistant, Department of Oral Medicine and Surgery, Faculty of Dentistry, Istanbul University, Istanbul, Turkey

${ }^{3}$ Professor, Unit of Oral Pathology, Oral Medicine and Laser-assisted Oral Surgery, Section of Odontostomatology, Department of Otolaryngological/ Dental/Ophthalmological and Cervico-Facial Sciences, University of Parma, Italy

${ }^{4}$ Research Assistant, Department of Pathology, Istanbul Faculty of Medicine, Istanbul University, Turkey

${ }^{5}$ Professor, Department of Oral Medicine and Surgery, Faculty of Dentistry, Istanbul University, Istanbul, Turkey

${ }^{6}$ Consultant Professor, Unit of Oral Pathology, Oral Medicine and Laser-assisted Oral Surgery, Section of Odontostomatology, Department of Otolaryngological/Dental/Ophthalmological and Cervico-Facial Sciences, University of Parma, Italy
\end{abstract}

Correspondence:

Istanbul University,

Faculty of Dentistry,

Department of Oral Medicine and Surgery

34393 Capa, Istanbul, Turkey

sertanergun@gmail.com

Received: 07-01-2010

Accepted: $23-04-2010$

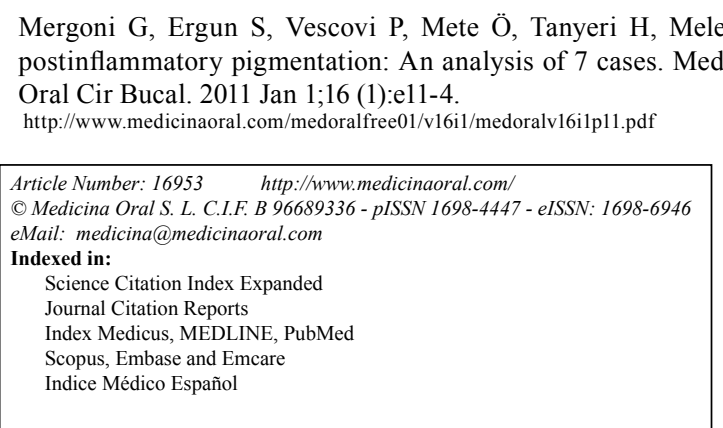

\begin{abstract}
Oral postinflammatory pigmentation (OPP) is a discoloration of the oral mucosa caused by an excess of melanin production and deposition within the basal layer of the epithelium and connective tissue of areas affected by chronic inflammation. Therefore, it is mandatory to demonstrate the association with a previous or concomitant inflammatory process in the same area of oral mucosa. Clinically OPP appears as a localized or diffuse, black to brown pigmentation. OPP may persist for many years even though the disappearing of the pigmentation after the resolution of the inflammatory state has been reported. We reviewed retrospectively the medical records and, when performed, biopsy examinations of 7 cases of OPP. Four cases were associated with oral lichen planus, two cases with lichenoid lesions and one case with proliferative verrucous leukoplakia. Despite a possible high prevalence of OPP, only a few reports concerning diagnosis, etiopathogenesis and clinical manifestation have been published so far.
\end{abstract}

Key words: Oral postinflammatory pigmentation, melanin, oral lichen planus.

\section{Introduction}

Oral postinflammatory pigmentation (OPP) represents a change in colour of the oral mucosa occasionally associated with chronic inflammatory disorders such as oral lichen planus (OLP) and other oral lichenoid lesions (OLLs), pemphigus, pemphigoid, periodontal disease, Steven-Johnson syndrome and graft versus host disease (1-3).
OPP is caused by an excess of melanin production and deposition within the basal layer of the epithelium and connective tissue of areas affected by chronic inflammation (3).

There are few specific data about the epidemiology of OPP. Murti et al. reported that $11 \%$ of 99 patients affected by OLP presented oral pigmentation (1). Apparently OPP is more common in dark-skinned patients (4). 
The pathogenesis of OPP has been little investigated. Few evidences, mainly extrapolated from dermatological studies, support the hypothesis of a stimulating effect of inflammation on melanocytes (3). No reliable criteria for diagnosis of OPP have been provided. It seems however mandatory to demonstrate the association with a previous or concomitant inflammatory process in the same area of oral mucosa.

Clinically OPP appears as a localized or diffuse, black to brown pigmentation. OPP may persist for many years even though the disappearing of the pigmentation after the resolution of the inflammatory state has been reported (5). Treatment is usually not necessary.

Here we report a clinicopathological evaluation of 7 cases of OPP altogether with a discussion on its possible etiopathogenesis and diagnosis. All the patients were observed at the Unit of Oral Pathology, Oral Medicine and Laser-assisted Oral Surgery of the University of Parma, Italy and at the Department of Oral Medicine and Surgery, Faculty of Dentistry, Istanbul University, Istanbul, Turkey.

\section{Case Reports}

\section{Case 1}

A 60 -years-old non smoker white woman was referred presenting a mucocutaneuos lichen planus. The histo- pathological report from skin biopsy supported the diagnosis of lichen. Skin evaluation disclosed the presence of erosive, ulcerative and crusty-like lesions surrounded by scar tissue on legs and shoulders. The examination of oral cavity revealed the presence of ulcerative, bilateral lesions on cheek mucosa as well as erosive, crustylike lesions on her lower lip (Fig. 1. A). Lesions were clinically compatible with OLP. Symptoms referred were pain and difficulties in eating. A biopsy on her left cheek mucosa was performed and the histopathological evaluation showed features suggestive of OLP. A complete regression of skin and oral lesions was obtained through systemic and local therapy with corticosteroids. A follow-up scheme of 1 visit every 3 months was scheduled. During controls a previously unnoticed pigmented lesion of left buccal mucosa was detected. This pigmentation was brown in colour and had a slight reticular pattern (Fig. 1. B). Clinical history and appearance could fit with OPP.

Case 2

A 58-years-old non smoker white man was referred for diffuse bilateral verrucous white lesions on his buccal mucosa and vestibular gingiva (Fig. 2. A). The histopathological examination of the biopsy specimen from his left buccal mucosa revealed epithelial hyperplasia with hyperkeratosis and presence of superficial band-
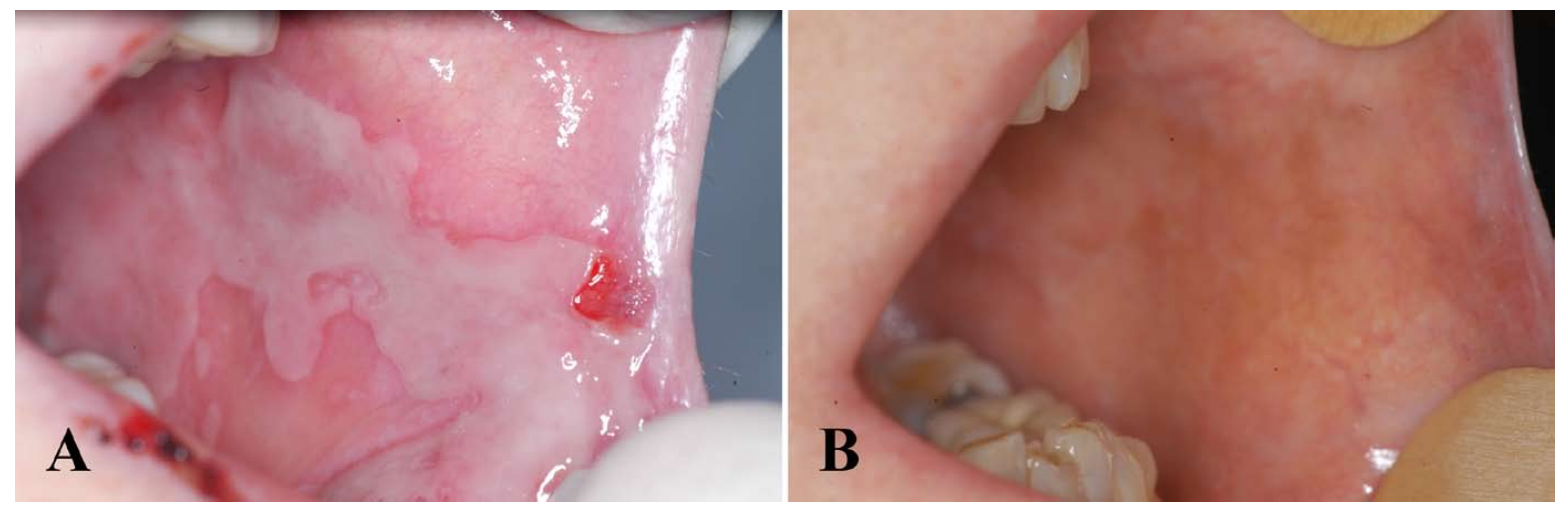

Fig 1. A. Ulcerative lesions involving buccal mucosa in a patient affected by OLP. B. OPP disclosed after inflammation resolution.
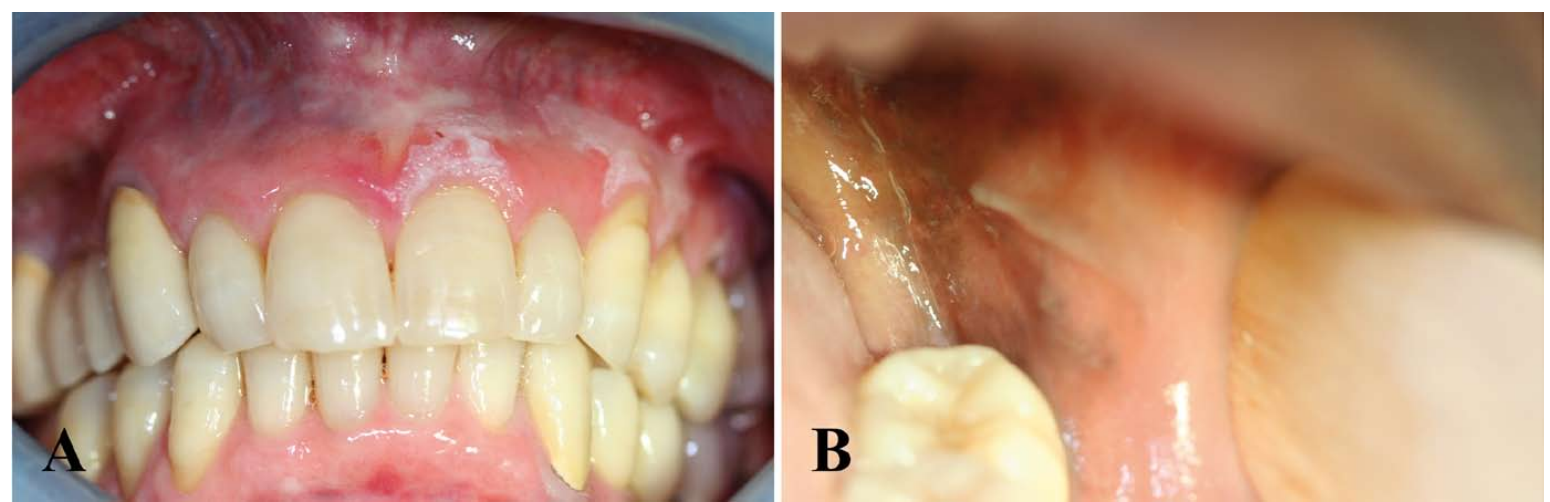

Fig 2. A. Diffuse Plaque-like lesions in course of proliferative verrucous leucoplakia. B. OPP of the retromolar pad in the same patient. 
like inflammatory lymphoplasmacytoid infiltrate in the connective tissue. The disorder was resistant to medical and surgical (laser vaporization) therapy. During 7 years follow up, the patient also experienced painful erosive lesions. During a clinical evaluation, darkening of buccal and vestibular mucosa bilaterally was noticed (Fig. 2. B). Pigmented lesions were diffuse and etiologic factors such as smoking or amalgam were excluded. Clinical and anamnestic findings were consistent with OPP in patient affected by proliferative verrucous leukoplakia (PVL).

Case 3

A 32-years-old non smoker white woman was referred because of the presence of erosive lesions on the hard and soft palate. These alterations have been present for 6 years. Pain, burning sensation and bleedings were reported. Clinical findings included diffuse, bilateral, irregularly bordered erosions on the palatal side of maxillary alveolar bone in association with small white papulae. A palatal biopsy has been taken three months before and the histopathological report was "lichenoid stomatitis". Topical therapy with corticosteroids was administered. Clinical examination after two months revealed the presence of an asymptomatic pigmented lesion on the right side of hard palate that was not present at the first evaluation. The lesion was dark to brown, with irregular margins and of about $1,5 \mathrm{~cm}$ of diameter. The clinical and anamnestic features were compatible with OPP.

Case 4

A 70-years-old non smoker white woman was affected by histopathologically proven OLP. The patient presented white reticular striae bilaterally on her cheek mucosa and plaque-like lesions on the dorsal surface of the tongue. A follow-up visit disclosed the presence of a dark to brown pigmented alteration with a reticular pattern on her left buccal mucosa. Such a finding could fit with the diagnosis of OPP.
Case 5

A 77-years-old non smoker white woman of was affected by reticular type of OLP. Wickham's striae were present on buccal mucosa bilaterally. On the left buccal mucosa was evident an asymptomatic brown pigmentation clinically compatible with OPP.

Case 6

A 50-years-old non smoker white woman was referred for bilateral lesions affecting her cheek mucosa. The lesions were present since one year. The intraoral examination revealed bilateral lichenoid alterations on her cheek mucosa in correspondence of metal-supported bridges. Brown pigmentations in the same areas were observed (Fig. 3. A). The allergy test to dental restorative materials was positive for nickel sulfate and palladium chloride. A biopsy was performed on her right buccal mucosa and the histopathological examination showed features suggestive of oral lichenoid lesions (OLL) that underwent histological regression. The presence of subepithelial melanophages, causing the clinically detectable pigmentation, was also demonstrated (Fig. 3. B). The clinical and histopathological features were consistent with OPP associated to OLLs.

\section{Case 7}

A 61-years-old non smoker white man was visited for bilateral plaque-like lesions in his retrocomisural mucosa. On the right side a pigmentation, irregular in color and margins, was observed. A biopsy was performed on his right buccal mucosa and the histopathological examination was compatible with OLP. The presence of subepithelial melanophages was also demonstrated.

\section{Discussion}

Despite a possible high prevalence of OPP, only a few reports concerning diagnosis, etiopathogenesis and clinical manifestation have been published so far (1-3, 5-8).

Melanocytes within the oral mucosa have been firstly identified in 1927, but their biological function is still

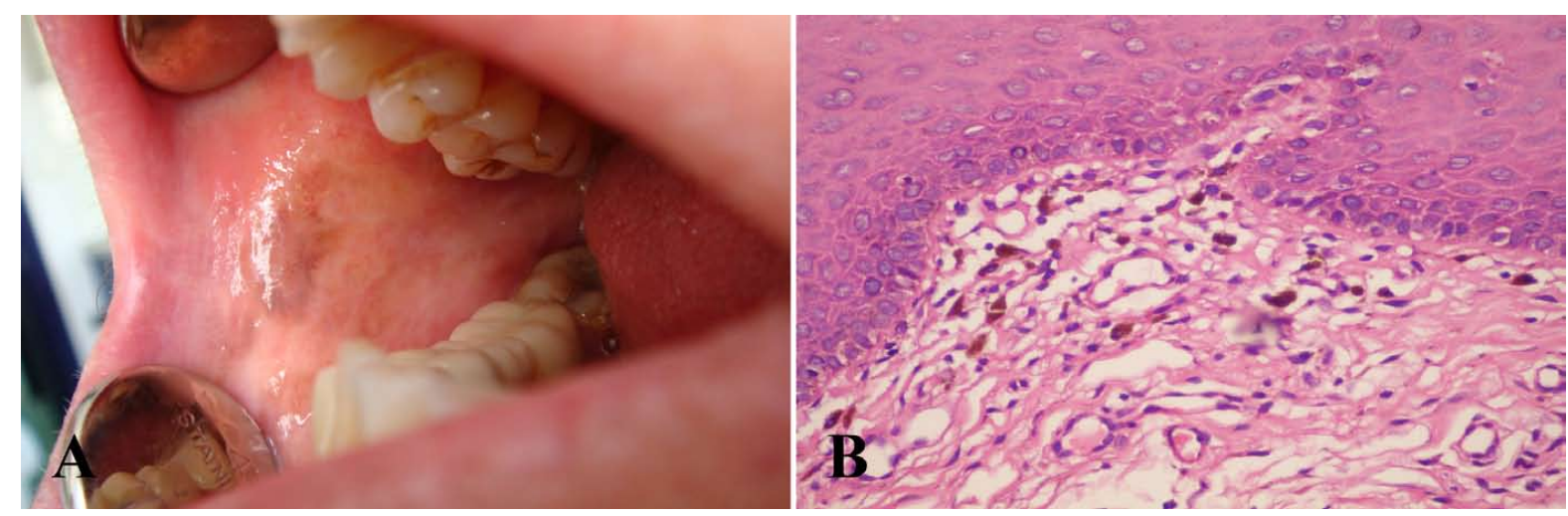

Fig 3. A. OPP associated with lichenoid lesions in correspondence of metallic restorations. B. Histopathological view showing the presence of subepithelial melanophages (H\&E, 40X). 
poorly understood (9). Presence of melanin granules within oral mucosa melanocytes has been reported in white-skinned individuals but it is usually insufficient to produce a clinically detectable pigmentation (9). Nevertheless, oral melanin production can be stimulated by several factors leading to oral pigmentation. The pathogenesis of postinflammatory pigmentation of the skin and of the oral mucosa may share some similarities. Therefore some considerations could be extrapolated from the dermatologic literature (9).

The development of a postinflammatory pigmentation includes two processes: an increase in melanin production and an abnormal distribution of the pigment (10). The first one is related to a direct stimulation of melanocytes by inflammatory mediators (Table 1). Papa and Kligman founded that a chronic inflammation of the skin induced by chemical irritation with sodium lauryl sulphate solution causes an increased density of melanocytes as well as enhanced enzymatic activity (11). The second step of the development of postinflammatory hyperpigmentation is an abnormal distribution of melanin pigment. A frequent finding in specimens taken from tissue affected by postinflammatory hyperpigmentation is the presence of macrophages laden with melanin (melanophages) in the connective tissue underlying epithelium (12). Probably melanin is released within the connective tissue where it is phagocytized by melanophages.

Four of the cases presented in this serie (1, 4, 5 and 7) represent OPP associated to OLP. Even cases of pigmented OLP seem to be a frequent finding, only a few have been reported $(1,7,8)$, As a matter of fact, Cooke in 1954 used the term "pigmented pattern" to classify this subtype of OLP (6). Stimulation of melanocytes by cytokines and inflammatory mediators released by subepithelial lymphocytic infiltrate may explain OPP in course of OLP. In case 5 the patient affected by OLP presents a pigmentation in strictly correspondence of the biting line. In this case it is possible that a melanocytic reaction was exacerbated also by traumatic irritations.

Patient described in case 2 had OPP associated to PVL. To the best of our knowledge this is the first case reporting such association. PVL is a rare subtype of oral leukoplakia characterised by slow growing, diffuse, verrucous lesions resistant to any treatment and associated with a high risk of malignant transformation (13). Similarly to OLP, a lymphocytic infiltrate within the superficial part of lamina propria is often present in PVLassociated lesions (14). This infiltrate may suggest that OPP in course of PVL and OLP could have the same pathogenesis.

While case 3 showed features of OLLs, case 6 exhibited mainly features suggestive of OLLs that underwent regression. Histopathologically, the lesions were characterized by a variable amount of subepithelial lichenoid lymphocytic infiltration and melanin pigment incontinence in the subepithelial area as a result of underlying basal layer degeneration.

In all except one case, OPP involved buccal mucosa. This site-specific distribution reflects the location of chronic inflammatory stimulus in course of OLP, OLL and PVL and possibly the high concentration of melanocytes in this oral subsite (9).

To date, no diagnostic criteria have been proposed for OPP. In our opinion, the diagnosis of OPP in a patient with a chronic inflammatory disorder presenting a pigmentation of the oral mucosa could be rendered only after the exclusion of the other pigmented lesions of the oral mucosa (including racial, tobacco and drugs related pigmentations). Additionally, it should be possible to demonstrate the association with a previous or concomitant inflammatory process in the same area of the oral mucosa.

\section{References}

References with links to Crossref - DOI

1. Murti PR, Bhonsle RB, Daftary DK, Mehta FS. Oral lichen planus associated with pigmentation. J Oral Med. 1979;34:23-4.

2. Sakai T, Sakai H, Hashimoto N, Hirayasu R. Gingival pigmentation beneath a metallic crown: light and electron microscopic observations and energy dispersive X-ray analysis. J Oral Pathol. 1988;17:409-15.

3. Patsakas A, Demetriou N, Angelopoulos A. Melanin pigmentation and inflammation in human gingiva. J Periodontol. 1981;52:701-4.

4. Kauzman A, Pavone M, Blanas N, Bradley G. Pigmented lesions of the oral cavity: review, differential diagnosis, and case presentations. J Can Dent Assoc. 2004;70:682-3.

5. James WD, Cooper KD, Todd RF 3rd, Brown C, Lewis D. Inflammatory acquired oral hyperpigmentation: association with melanophages demonstrating phenotypic characteristics of antigen presenting cells and activated monocytes. J Am Acad Dermatol. 1987;16:220-6.

6. Cooke BE. Leucoplakia buccalis and oral epithelial naevi; a clinical and histological study. Br J Dermatol. 1956;68:151-74.

7. Singh S. Violaceous hue in oral lesions of lichen planus. Int J Dermatol. 1994;33:603-4.

8. Takeuchi Y, Iwase N, Suzuki M, Tsuyuki S. Lichen planus with involvement of all twenty nails and the oral mucous membrane. J Dermatol. 2000;27:94-8.

9. Barrett AW, Scully C. Human oral mucosal melanocytes: a review. J Oral Pathol Med. 1994;23:97-103.

10. Morelli JG, Norris DA. Influence of inflammatory mediators and cytokines on human melanocyte function. J Invest Dermatol. 1993;100:191S-195S.

11. Papa CM, Kligman AM. The behavior of melanocytes in inflammation. J Invest Dermatol. 1965;45:465-73.

12. Nordlund JJ. Postinflammatory hyperpigmentation. Dermatol Clin. 1988;6:185-92.

13. Hansen LS, Olson JA, Silverman S Jr. Proliferative verrucous leukoplakia. A long-term study of thirty patients. Oral Surg Oral Med Oral Pathol. 1985;60:285-98.

14. Cabay RJ, Morton TH Jr, Epstein JB. Proliferative verrucous leukoplakia and its progression to oral carcinoma: a review of the literature. J Oral Pathol Med. 2007;36:255-61. 